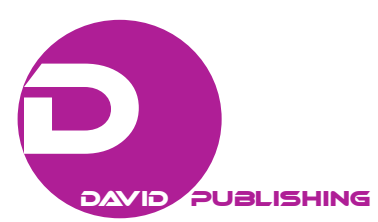

\title{
Geo-Spatial Information System for Developing Tourism Industry in Kandy District, Sri Lanka
}

\author{
T. M. S. P. K. Thennakoon \\ University of Sri Jayewardenepura, Gangodawila, Nugegoda, Sri Lanka \\ H. M. G. D. Welagedara \\ Strategic Enterprise Management Agency, Colombo, Sri Lanka
}

\begin{abstract}
Tourism is a rapidly growing investment point in Sri Lanka, where huge investment is takeing place. Even though the investment is very massive, the planning, development, and marketing are key components of success in tourism zone enhancement. The main objective of this study was to implement a geo-spatial information system for development of tourism in Kandy district. Primary data collection methods i.e. questionnaire survey, interviews, focus group interviews, and observations were employed for data collection. Google maps with Google API standards which are specially designed for developers and computer programmers were used for implementation of the system. System requirements were identified by interviewing tourists and observations made on tourist sites. Proximity analysis, spatial joint, and network analysis with Google direction application program interface (API) and Google place API were used to analyze data. The study highlights the potential tourist attractions and the accessibility and other required details through a web output. Issues and challenges faced by travelers are mainly lack of specific location information, public transport schedules, and reliable tourist attraction information. Online geo-spatial information system created in this study provides a guide for tourists to find the destination routes, the service areas, and all necessary details on particular destinations.
\end{abstract}

Keywords: tourism, geo-database, API, GIS, geo-spatial, Kandy

\section{Introduction}

Tourism is generally considered to be a positive agent in the economic and regional development process. Tourism has an influence on the economy of the country, on the natural and built environment, on local population at the destination and as well as for the tourists themselves.

National and local tourism policies as well as the necessary international agreements or other processes should be established, to approach effective tourism development, management, and monitoring (United Nations World Tourism Organization-UNWTO, 2015). Achieving sustainable tourism is a continuous process and it requires constant monitoring of impacts, introducing the necessary preventive and/or corrective measures whenever necessary sustainable tourism should also maintain a high level of tourist satisfaction and ensure a

T. M. S. P. K. Thennakoon, Ph.D., associate professor, Department of Geography, University of Sri Jayewardenepura, Gangodawila, Nugegoda, Sri Lanka.

H. M. G. D. Welagedara, M.Sc., Strategic Enterprise Management Agency, Colombo, Sri Lanka.

Correspondence concerning this article should be address to Prof. T. M. S. P. K. Thennakoon, Department of Geography, University of Sri Jayewardenepura, Gangodawila, Negegoda, Sri Lanka. 
meaningful experience to the tourists, raising their awareness about sustainability issues and promoting sustainable tourism practices amongst them (UNWTO, 2015).

According to the current tourism development strategy in Sri Lanka, the main target of tourism sector for next five years is to achieve a target number of 2.5 million tourists by 2016. The master plan strategy developed by Ministry of Economic Development predicts tourist's arrivals to Sri Lanka up to 2020, also it gives a classification of tourism by region (Sri Lanka Tourism Development Authority_SLTDA, 2015). According to the strategic development plan, tourism is rapidly growing investment in Sri Lanka, where huge investment is takeing place. Even though the investment is very massive, the planning, development, and marketing are key components of success in tourism zone enhancement.

In tourism industry time is one of main factors which influence tourists for their travel plan. Kandy is where set of historical tourism places and events occur therefore according to the time frame planning should be done. Manageing the time and planning for a better tour is a challenge with printed materials such as guide books. Traditional papers based tourist maps are time-consuming for reading. When referring a traditional map, it requires much time to find a specific destination compared to internet based maps. Searching places from internet saves time rather than referring a page of a book, because user can search it by keywords. Online information plays a major role in the world. However, digital information is inadequate for tourism industry in Sri Lanka. Acquiring comprehensive digital information based on tourism infrastructural facilities is a great challenge for tourist. With this vacuum, this study was specifically geared to develop a geo-spatial information system for tourism development in Kandy District. The secondary objectives of the study were to identify the issues and challenges in existing tourism system, identify the existing potential tourist attractive destinations in Kandy district, and develop a tool to search route information within destinations and closest facilities, service areas, accommodation, banks, shopping malls, and food within the selected areas.

\section{Literature Review}

Researches undertaken related to application of GIS in identification optimum path for tourist places are limited in Sri Lanka. However, limited number of literature can be ascertained at global level. The research undertaken by Gill and Bharath (2013) on identification of optimum path for tourist places using GIS based network analysis was important. The aim of this paper was to explore the GIS based Network analysis for rout optimization of tourist places in Delhi city. The study determines the optimal route for the tourist origin to destination places including visiting time at each tourist destination (Gill \& Bharath, 2013).

Tourism planning is not only about determining the optimum route even though Gill and Bharath (2013) consider only destination routing, service areas, and facilities are more important when planning a tour. Tourist wants to determine accommodation, restaurants, banks, ATMs, shopping malls, hospitals, cinemas etc. Facilities and services areas are not addressed in this study, it only considers visiting different tourist places as priorities. Main priority is given to road network; providing a road network by a GIS based network analysis is better application rather than referring printed maps but when it comes to tourism there are factors to be considered. Tourist is an adventive person to that area, they are not aware of the particular area. Therefore, in-detail guide map should be provided for them. Infrastructure facilities are more important therefore to achieve sustainable tourism development better to locate service areas and facilities along with road network.

Application of GIS for promotion, planning, and development of tourism in Kandy district of Sri Lanka is very important because there are many cultural, modern, and ecological destinations which are still hidden 
(Udawattakelle forest, Lankathilaka and Gadaladeniya temples etc.) for the public. Even though there are many tourist attractions in Kandy district, few of them are popular. Tourism is bound to Kandy city limits, therefore categorizing and positioning the hidden tourism destination in an appropriate manner are very important to promote them as new tourism destination. Multi-media objects perform a major role in promotion process text, graphics, animation, and video, they can help to enhance the value of the destination, using a multi-media database is the best opportunity for sustainable tourism development.

\section{Research Methods}

Kandy district of Sri Lanka was selected as the study area which is the last capital of the Sinhala Monarchy. Kandy City is an international religious center and central place which links to many tourism destinations in Sri Lanka. It is also one of UNESCO's World Cultural Heritage sites since 1988. Primary data collection methods i.e. questionnaire survey, interviews, focus group interviews, and observations were employed for data collection. Google maps with Google API standards which are specially designed for developers and computer programmers were used for implementation of the system. Depending on Google server data, the tools were designed and developed. Strategic tools (Google place API and Google Direction API) offered by Google are used.

\section{Design Process (Web GIS Development Cycle)}

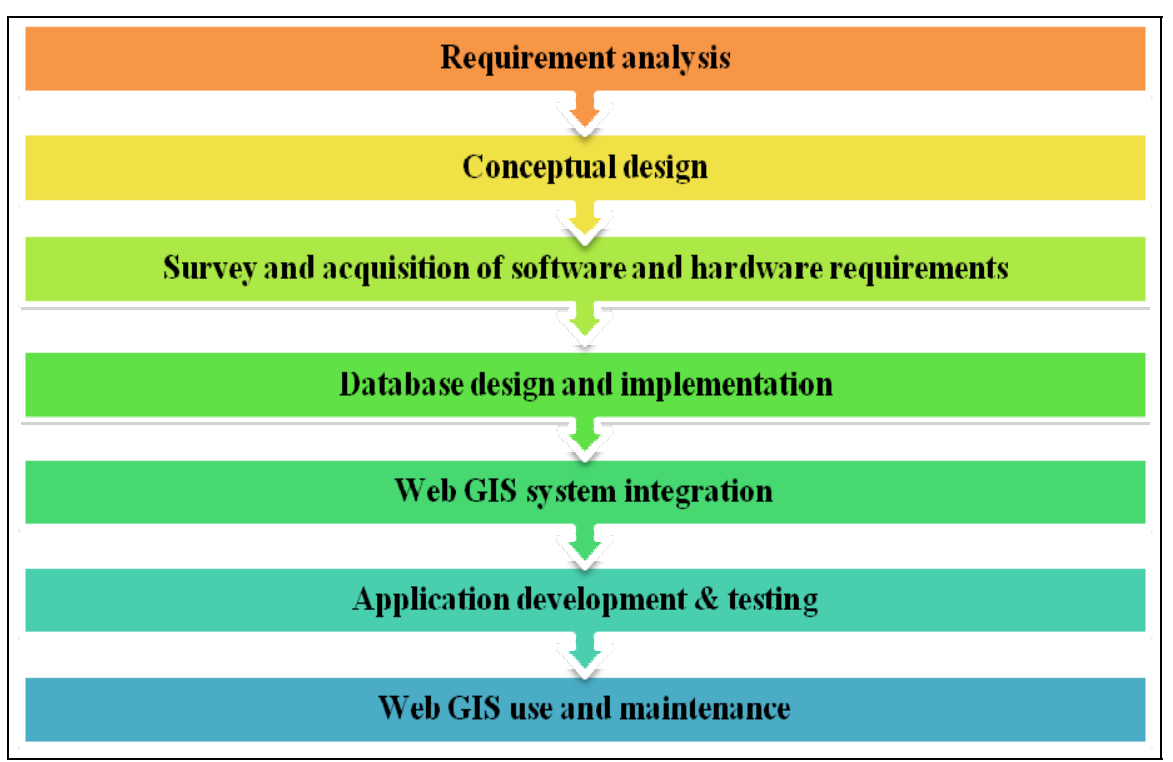

Source: Created for research based on Alesheikha, Helali, and Behroz (2000).

\section{Data Analysis}

Development of the geospatial information system allows users to access spatial data via a web browser interface. System users will interact with web server and spatial database to retrieve the relevant required information.

Proximity analysis is a process of answering distance related questions, for example:

- How close is this tourist destination to main road?

- Do any roads pass within 100 meters of a hotel?

- What is the distance between two locations? 
- What is the nearest or farthest feature from something?

- What is the distance between each feature in a layer and the features in another layer?

- What is the shortest street network route from some location to another?

Multi-ring buffer tool has been used to classify the areas around a feature into near, moderate, and long distance classes for road analysis. Buffer and multiple ring buffer create area features at a specified distance around the input features. Network Analyst can determine the best sequence to visit the locations when it considers more than one location (Arc GIS Resource Center, 2015).

In this study road and railway distance buffers are created to identify the closest destinations to the main roads and railway network analysis is one of the GIS based data analysis techniques used in this study. Using network analysis, a user will be able to identify the hotels, shopping malls, ATMs, banks etc. around a specific a destination. Tourist can plan routes for their entire city trip, calculate drive-times, locate facilities and service areas where tourist can locate (accommodation, restaurants, banks, shopping malls, hospitals etc.)

\section{Initial Steps of Creating Web}

Place searches return a list of places based on a user's location or search string. Place details requests return more detailed information about a specific tourist attraction, including user reviews. Place auto-complete can be used to automatically fill in the address of a tourist attraction as user type. Each of the services is accessed as an HTTP request, and returns either a JSON or XML response. All requests to a place service must use the HTTP protocol, and include an API key. The Google Places API web service uses a place ID to uniquely identify a place.

The Google Places Web Service is a service that returns information about a "place" as establishment, a geographic location, or prominent point of interest using an HTTP request. Two basic Place requests are available: a place search request and a place details request. Place search returns the coordinates of the location and details are provided by place detail request. The Google Places API web service is a service that returns information about places defined within this API as establishments, geographic locations, or prominent points of interest using HTTP requests (Google Maps APIs for Web Developers, 2015).

The directions service object communicates with the Google Maps API which receives direction requests and returns computed results. The directions service can return multi-part directions using a series of waypoints. Directions are displayed as a polyline drawing the route on a map and the instruction can be given as statements (Google map API Direction API, 2015).

\section{Generating a Key}

All JavaScript API applications require authentication using an API key. Including a key in your request allows you to monitor your application's API usage in the Google developers console, enables per-key instead of per-IP-address quota limits, and ensures that Google can contact you about your application if necessary. To use the standard API, you need a browser key (https://developers.google.com/maps/web/). The steps to acquire an API key are to enter the Google developers console and create or select a project click to continue to enable the API.

Here the API Credentials and the API key will be generated. The generated key is displayed to use Google API. The generated key should copy and paste within the html and JavaScript code. After pasting the key on "your API key" space the developer is able to access Google map API's and use the map functionalities to develop new tools. 


\section{Creating the Server}

Apache Tomcat Server version 2.0 was downloaded and installed by setting the port number for local host. Server was configured and checked by opening in browser with defined port number (http://localhost:8080/). Apache Version: 2.2.8 and PHP Version: 5.2.6 are used.

\section{Research Results \& Discussion}

Analysis was undertaken based on the information gathered by the researchers and the base layers obtained from survey department Sri Lanka. The problems faced by the tourists were carefully considered. Comments of the stakeholders were also provided a working frame for the identified solution as the functionality of the implementation.

\section{Existing Issues and Challenges in the Tourism System}

Several challenges and issues exist in tourism industry in Sri Lanka. The study ascertained that there were no existing statistical figures obtainable from government on issues and views of tourists, therefore the researchers conducted several primary data collection techniques. Most of the tourists prefer to use an information system to optimize the travelling activities and they are dissatisfied with the current information hubs and tourist maps available.

When implementing a system for tourism, the user's view of a particular scenario is important and therefore this study focused on neediness of preference of an information system to optimize the tourist travelling activities. The analyzed data proved that almost $82 \%$ of tourists prefer an information system to optimize the travelling activities while $18 \%$ do not prefer an information system. In an initial stage of requirement of gathering for a system, the tourists satisfaction on existing systems is more beneficial and disappointments are the implementation, points of a new system interpret the tourist satisfaction of existing tourist printed maps and information hubs. The study indicated that more than $50 \%$ of tourists are dissatisfied with the current information processes and information hubs while $27 \%$ satisfied and $23 \%$ have no clear perception.

Places of storing the location data are more important once it comes to travelling because tourism is all about location. Tourists cannot keep the all locational details in their minds, since a tourist is new comer to the destination. Thus, study focused on the above research question and ascertained that tourists use different types of methods to store the tourist attraction details as indicated in Figure 1.

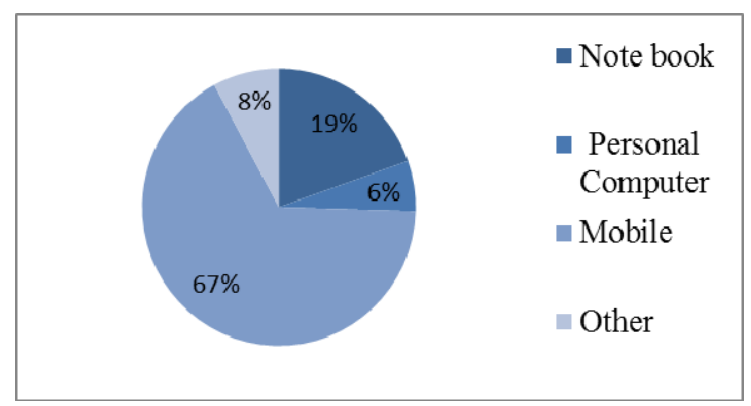

Figure 1. Methods of storing tourists attraction details.

According to Figure 1, 67\% of tourists use mobile devices to store the location data while $8 \%$ store these details on their note books and $6 \%$ on personal computers. More than $40 \%$ of the location data are stored as 
digital maps. Mainly they use offline maps where they can use without internet connection, because in Kandy the free Wi-Fi hot spots are not available. Nearly 25\% use the paper-maps, about 20\% use a notebook for their reference. Approximately $80 \%$ of tourists use Google maps as their travel guide while a very few tourists $5 \%$ use travelling application like lonely planet.

The time consumption to locate a destination is important factor since tourists have a shorter period to visit all the tourists' attractions in Sri Lanka. This was significantly considered by the study and the summarized time was taken to locate a place for tourist.

Most of the tourists make attempt to find a location and the destination details within 10-15 minutes. Nearly $40 \%$ of tourists take more than that to find a destination and to locate the place. As per the discussions had with them it is noted that this process was easier for them because of Google maps and other mobile applications. Reason for not using local mobile network connections is the high internet access cost. Majority of tourists use hotel Wi-Fi connections for internet access and they prefer Wi-Fi Hot Spots in city limits to find the locations through internet base maps, since they do not have a respectable updated travel guide.

The study also found the frequency of seeking directions by tourists i.e. nearly $50 \%$ of tourists have asked for direction from locals more than twice, since they are new to the destination and have less awareness about the location. Nearly $20 \%$ of tourists were practiced to travel alone while $80 \%$ of tourists run through asking directions from locals. The reason for not asking direction was lack of reliability and distrust of local people and sellers.

Tourists face difficulties of finding bus schedules and routes from their destination to tourist attractions. Lack of transport information is the main problem. Even though there are buses available, the routes are not specified for tourist attractions and proper schedules are not officially published in the internet (e.g. Kandy railway station).

Even though there are many sites named as tourist attractions in Kandy district, few of which were top listed in tourists visit. There were many reasons such as time, distance, transportations mode, map location, and popularity of the place which have contributed to it. According to the interviews conducted with tourists in Kandy the main attractions are Peradeniya Royal Botanical Garden and the Temple of Tooth. Identify the existing potential location around the main attraction is a respectable method to promote the destination as visitors already visited the most standard locations, if tourists required time they will visit the noted location as well. There should be an upright promotion point including a map with details on locations for designing this.

Time is the most important factor that people try to find the best and the easiest way to travel to a place and which requires knowledge on exact route. Avoidance of traffic is much important in the effective transportation. The study created a traffic map layer by overlaying with the direction map which is more covenient and easy to locate a place or to find an alternative route by passing traffic. Finally, the study created a digital application including tourists requirements in one output. Using this application, tourists will be able to locate hotels, resorts, restaurants, ATM, banks, hospitals, coffee shops likewise the search results can be customized accordingly (Figures 2-4).

Overlay of road buffer gives a picture of accessibility together it provides a land cover representation. Principally topmost tourist attractions are within the $500 \mathrm{~m}$ distance to main roads in Kandy city area and there are locations which can be visited among the city tour. The tourist attraction scattered around the main roads, it is a massive promotion point to tourist attraction reflected to accessibility. 


\section{Developing Tool to Find the Routes Within Destinations and Closest Facilities}

When considering the questionnaire survey, almost all the tourists are familiar with Google maps, some of whom use offline maps. Therefore, developing a tool based on Google API's is beneficial for users because of being familiar with the Google maps, every person is aware of and trusts the source. Even though the product (tool, software) quality is high the travels all ways try to use a reliable source as their guides. As they are new to the regions they depend on a trusted source similar to Google, download offline maps or application comparable lonely planet.

Considering the reliability and faith prominent well known source is advantageous for tourist since they are new to the region it's not $100 \%$ accurate but capable of location where the right place is. When considering this tool most important functionality is locating a tourist attraction destination for that data retrieved from the collected database which is more accurate. Since the travels are now aware about the location more accurately location information is important therefore that location information is retrieved from the MySQL database through a PHP query with location description images etc.

\section{Discover the Routes and Directions Between Destinations With Google API}

Direction API is used to identify the routes between two destinations. This tool can be accessed for anyone from anywhere in the world who knows the tool URL. With the direction of API there are several functions that combine to give an appropriate output and the layers useful for travels like traffic map.

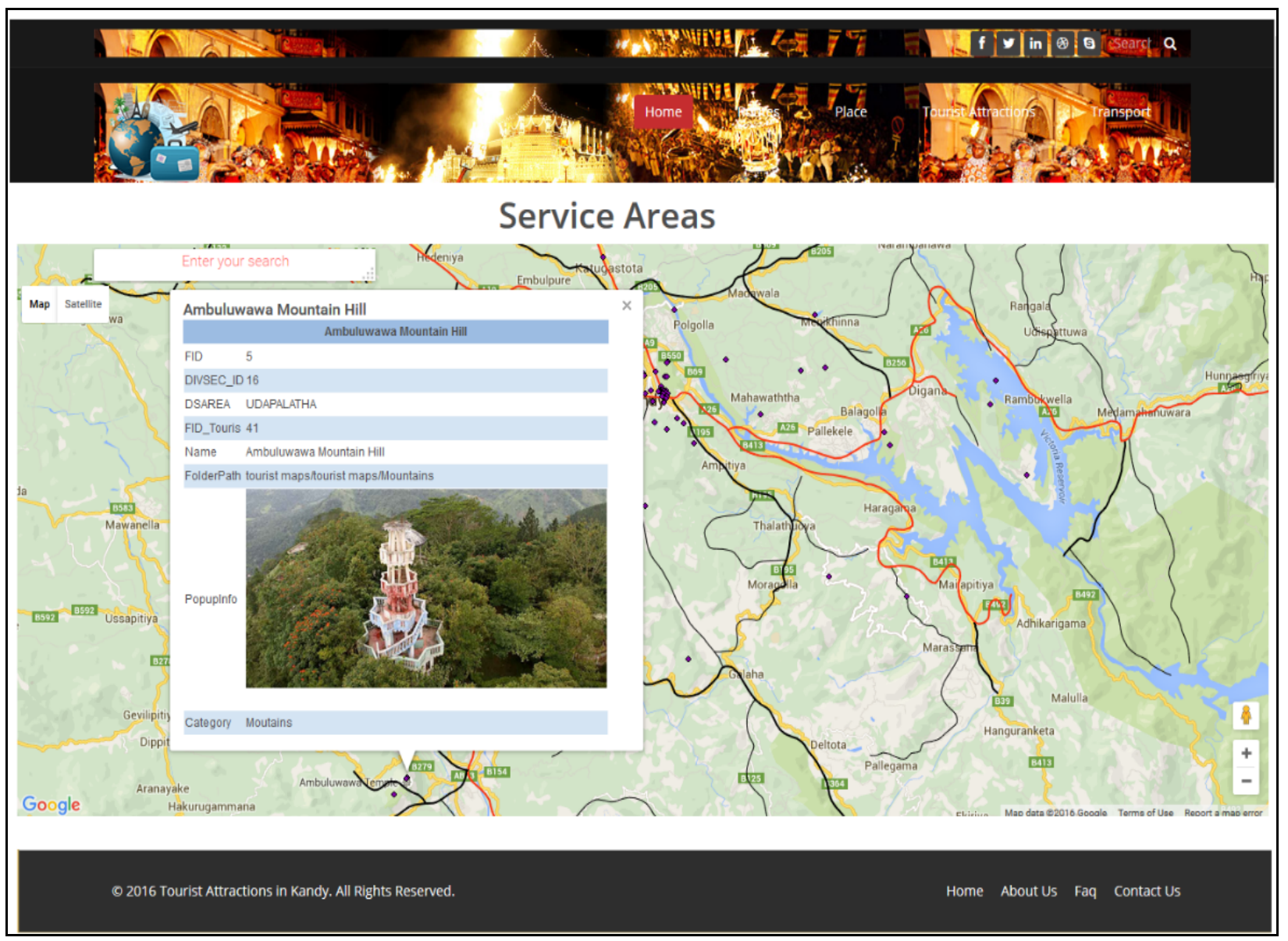

Figure 2. Tourist attraction details proceeding $\mathrm{kml}$ on Google maps. 


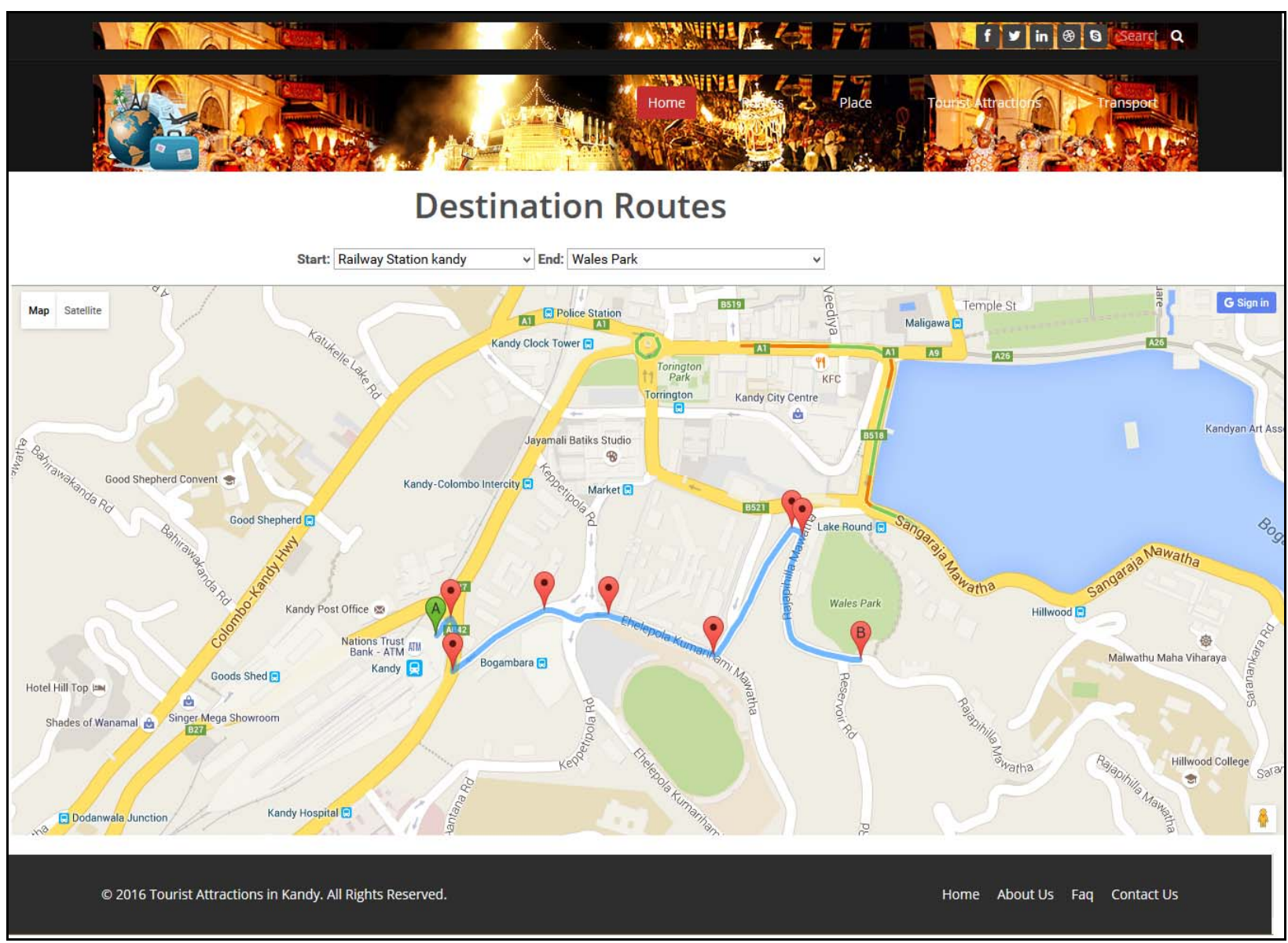

Figure 3. Route solution map within two destinations source.

The potential tourist attraction locations are listed in combo box and it acquires input as selected options, locations are customized accordingly. Place ID's are used as inputs then the route is present with the two locations, the result will be displayed in the map with directions distance and the turning points. The main places are listed on the start side (main bus stand, railway station, and key land marks) the user is able to select the known starting point, the map is customized to get direction from a known land mark to a tourist attraction destination, if a tourist droved to Kandy by train and if he/she wants to go to Temple of Tooth.

Then it displays the route between the initial start and end selections on the map as Figures 4 and 5 demonstrate. The request is taken from the change event of the combo box when the combo box value chages it calcultes the route, listens to change events from the start and end lists. When it comes to the functionality of direction service first it , any existing markers should be removed from the map.

Then it retrieves the start and end locations and creates a directions request using walking directions. The route of the directions passes the response to a function to create markers for each step change. For each step, place a marker, and add the text to the marker's info window. Also attach the marker to an array so function can keep track of it and remove it when calculating new routes. Finally it opens an info window when the marker is clicked on, containing the text of the step.

Nowadays time is more important therefore people try to find the best and the easiest way to go to a place. When condiering the effective way traffic is more important there for the traffic map layer which is also 
overlayed with the direction map which is more covenient and easy to locate a palce or to find an alternative route by passing traffic.

Google traffic maps introduce colors and the colors show the speed of traffic on the road.

- Green: No traffic delays.

- Orange: Medium amount of traffic.

- Red: Traffic delay.

The darker the red, the slower the speed of traffic on the road. Gray or blue lines on the map show the routes available for the user.

Traffic incidents also display on traffic maps, traffic incidents include these types of delays. To see details about what happened, user can click on the icon displayed on top of the route map over traffic layer.

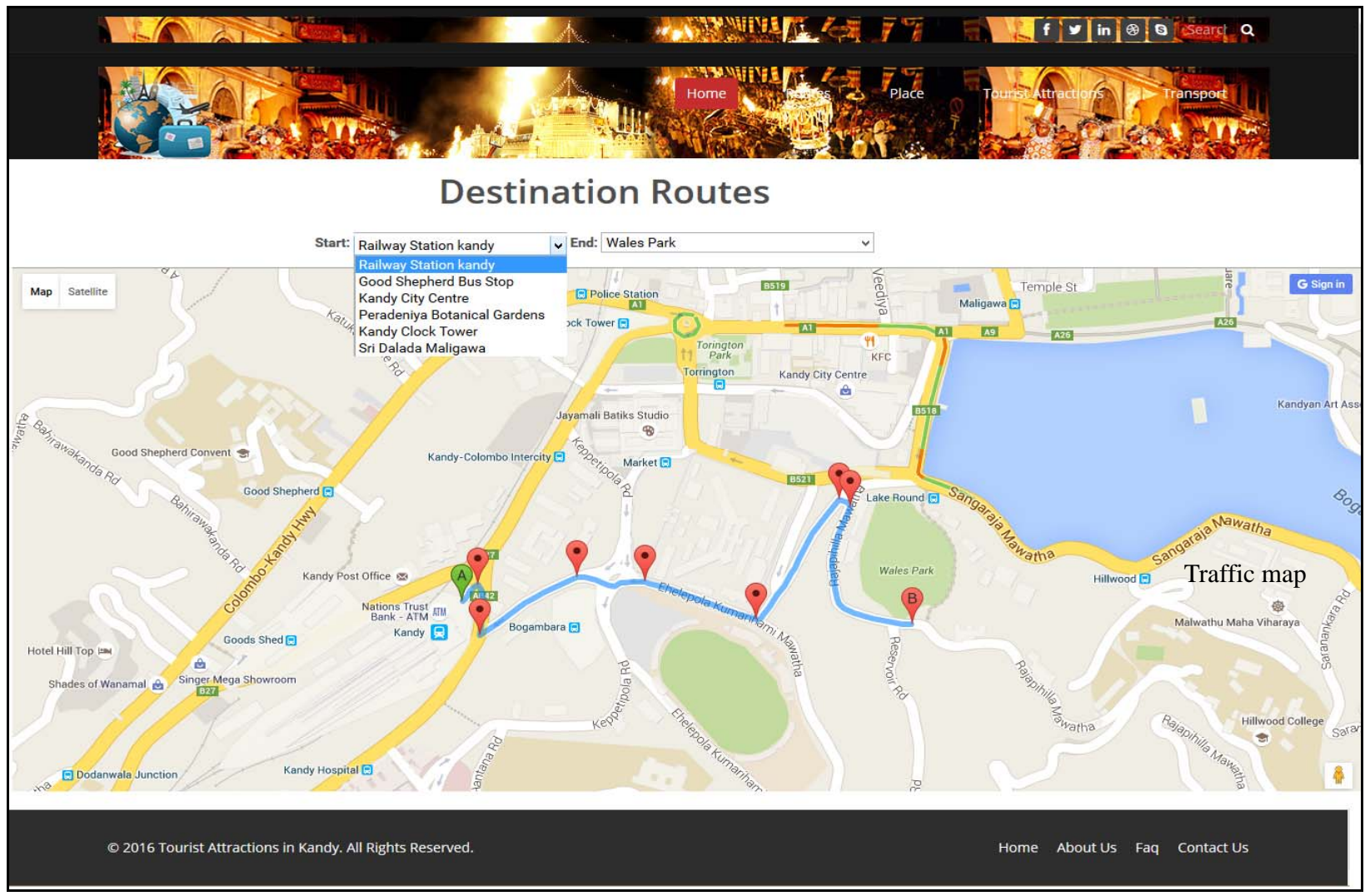

Figure 4. Traffic layer with route display.

Discover the Accommodation, Banks, Shopping Malls, Food Within the Selected Areas With Google API

Map contains a text area to search locations and facilities, user can search by key words (Figure 5) like hotels, resorts, restaurants, ATM, bank, hospitals, coffee shops likewise the search results can be customized accordingly.

After entering the searching of key word the associated resources will appear on the selected map area. The map is considered simple about the zoom map area. User has to zoom to the necessity level then enter the specific key word. When user zooms out it will show up further resources.

As per the below Figure 6 it shows the ATM search result in Kandy city limits. As following by steps first user will enter the searching of key word ATM and the user should require area by zooming to the location 
there after the required result will be shown on the map. This map layer overlays the tourist attraction locations in excess of service area details.

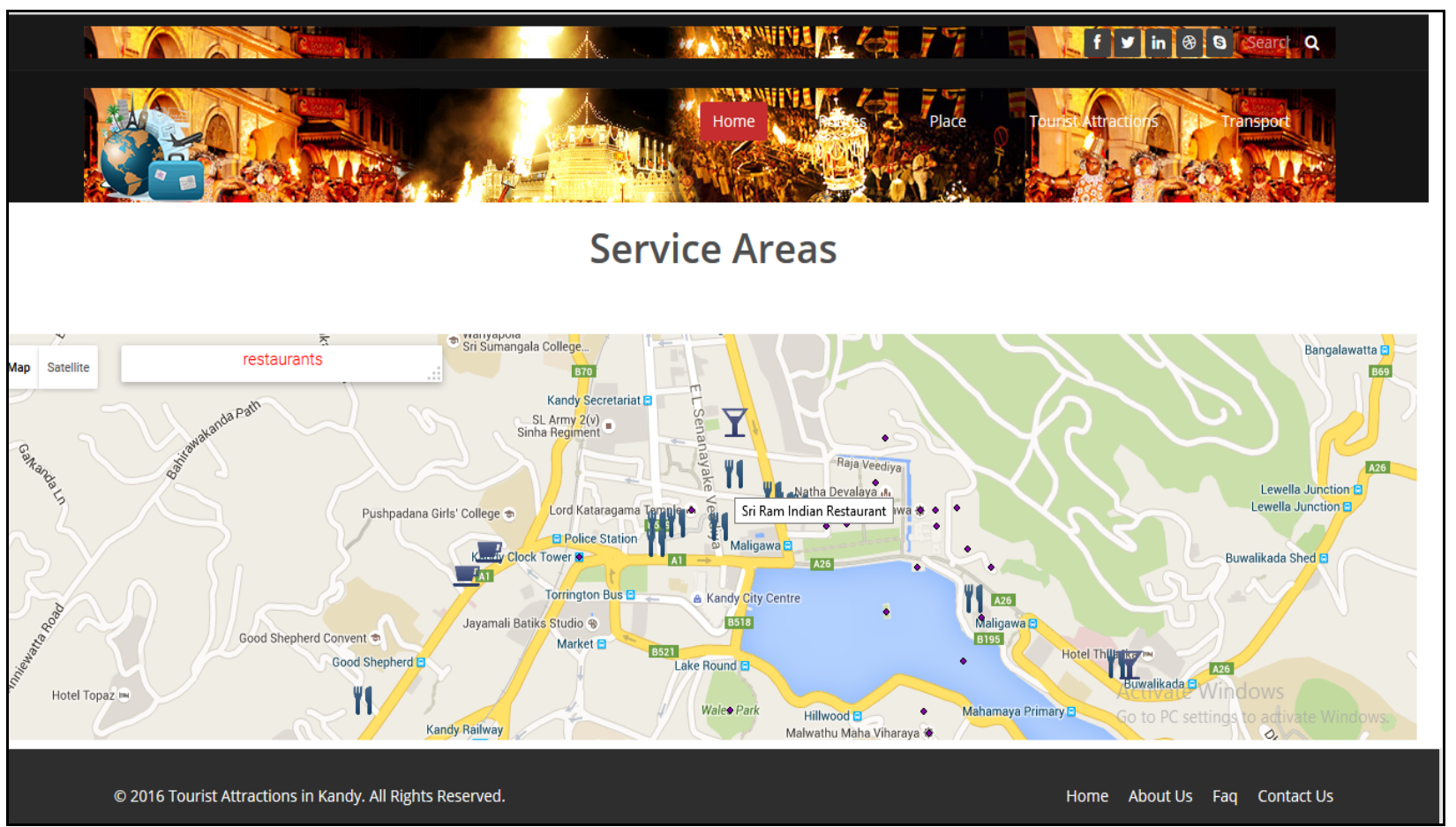

Figure 5. Hotel search output over destinations.

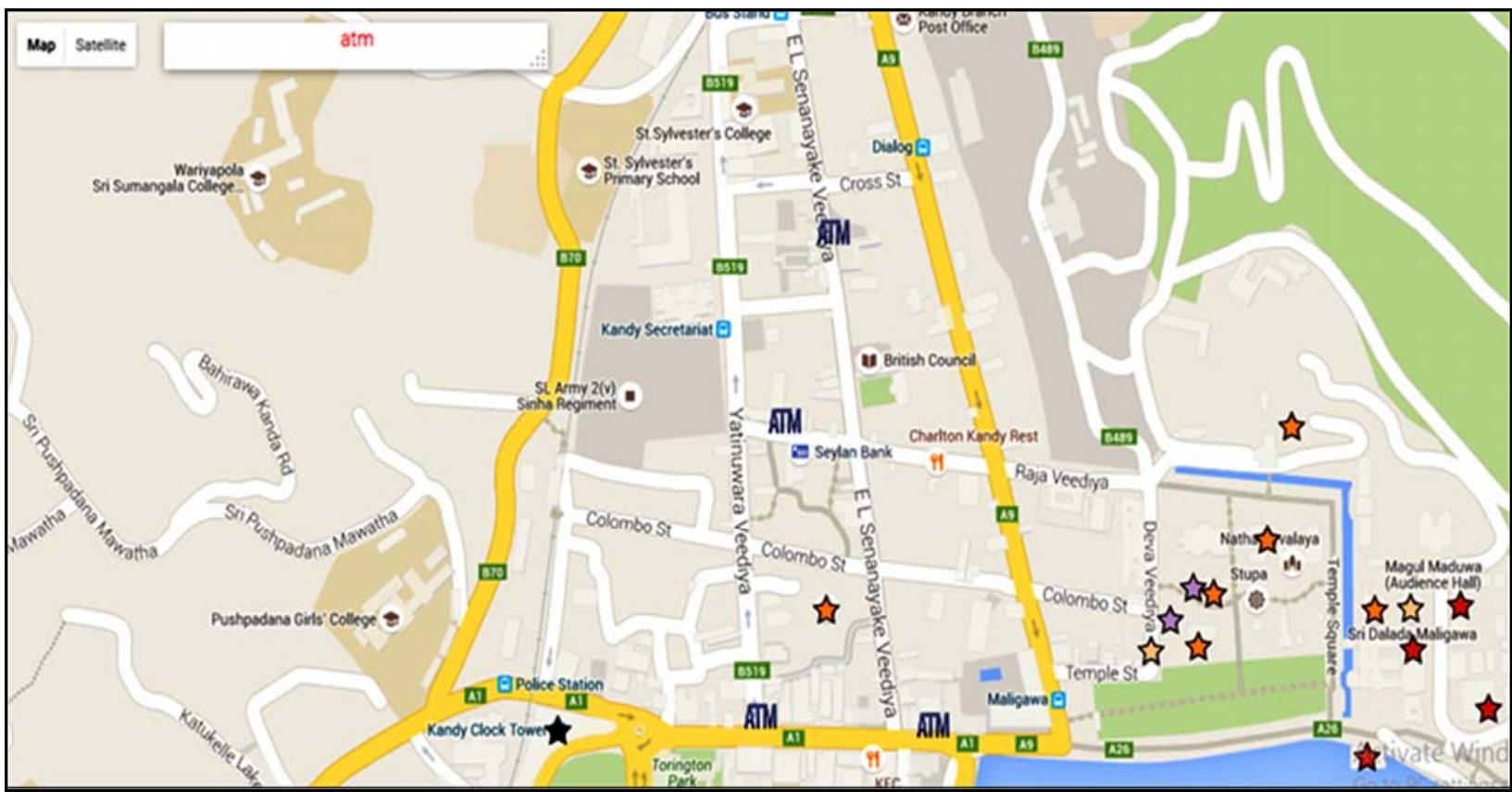

Figure 6. ATM search result in Kandy city.

Finally the development of the tool to find the routes within destinations and closest facilities, service areas, accommodation, banks, shopping malls, food within the selected areas is combined with the Google APIs. 


\section{Conclusions}

Guide information and tour package information can be displayed as a promotion factor on the web site. As per the survey, more than $60 \%$ of the tourists store the collected location data in their mobile devices and others use a note book to store data. Therefore, mobile applications are more popular also user friendly. Summary of the results indicates what the user expects. From the derived information, it was evident that many of the younger tourists were active with the role of information systems with modern global systems and applications. Most of the tourists' tradition is common platform of location identification rather than a specific application. Standard Google maps are used to locate a previously identified destination. The typical Google map gives generalized information about locations. Therefore, customized maps are further advantageous and also it contributes to acquiring a perfect picture and even a new tourist motivation to use the maps. If tourists do not have smart phone, the device can be promoted to purchase with tourist digital information package at the airport or in main tourist information centers with a deposit and the tourist can return it when they leave the country.

\section{References}

Alesheikha, H., Helali, H., \& Behroz. (2015). Web GIS: Technologies and its applications. Retrieved from http://www.isprs.org Arc GIS Resource Center. (2015). Network analysis. Retrieved from http://help.arcgis.com

Gilli, N., \& Bharath, B. D. (2013). Identification of optimum path for tourist places using GIS based network analysis: A case study of New Delhi. International Journal of Advancement in Remote Sensing, GIS and Geography, 1, 34-38.

Google Maps APIs for Web Developers. (2015). Retrieved from https://developers.google.com/maps/web/

Sri Lanka Tourism Development Authority. (2015). Developing \& planning the markets tourism development strategy. Retrieved from: http://www.sltda.lk

United Nations World Tourism Organization. (2015). Sustainable development of tourism. Retrieved from http://sdt.unwto.org/content/about-us-5 